\title{
The McDonald Observatory search for pulsating sdA stars
}

\section{Asteroseismic support for multiple populations ${ }^{\star}$}

\author{
K. J. Bell ${ }^{1,2}$, I. Pelisoli ${ }^{3}$, S. O. Kepler ${ }^{3}$, W. R. Brown ${ }^{4}$, D. E. Winget ${ }^{5,6}$, K. I. Winget ${ }^{5,6}$, Z. Vanderbosch ${ }^{5,6}$, \\ B. G. Castanheira ${ }^{5,6,7}$, J. J. Hermes ${ }^{8, \star \star}$, M. H. Montgomery ${ }^{5,6}$, and D. Koester ${ }^{9}$ \\ 1 Max-Planck-Institut für Sonnensystemforschung (MPS), Justus-von-Liebig-Weg 3, 37077 Göttingen, Germany \\ e-mail: bell@mps.mpg.de \\ 2 Department of Physics and Astronomy, Stellar Astrophysics Centre, Aarhus University, Ny Munkegade 120, 8000 Aarhus C, \\ Denmark \\ 3 Instituto de Física, Universidade Federal do Rio Grande do Sul, 91501-970 Porto-Alegre, RS, Brazil \\ 4 Smithsonian Astrophysical Observatory, Cambridge, MA 02138, USA \\ 5 Department of Astronomy, University of Texas at Austin, Austin, TX 78712, USA \\ ${ }^{6}$ McDonald Observatory, Fort Davis, TX 79734, USA \\ 7 Baylor University, Waco, TX 76798, USA \\ 8 Department of Physics and Astronomy, University of North Carolina, Chapel Hill, NC 27599, USA \\ 9 Institut für Theoretische Physik und Astrophysik, Universität Kiel, 24098 Kiel, Germany
}

Received 22 April 2018 / Accepted 26 May 2018

\begin{abstract}
Context. The nature of the recently identified "sdA" spectroscopic class of stars is not well understood. The thousands of known sdAs have H-dominated spectra, spectroscopic surface gravity values between main sequence stars and isolated white dwarfs, and effective temperatures below the lower limit for He-burning subdwarfs. Most are likely products of binary stellar evolution, whether extremely low-mass white dwarfs and their precursors or blue stragglers in the halo.

Aims. Stellar eigenfrequencies revealed through time series photometry of pulsating stars sensitively probe stellar structural properties. The properties of pulsations exhibited by sdA stars would contribute substantially to our developing understanding of this class. Methods. We extend our photometric campaign to discover pulsating extremely low-mass white dwarfs from the McDonald Observatory to target sdA stars classified from SDSS spectra. We also obtain follow-up time series spectroscopy to search for binary signatures from four new pulsators.

Results. Out of $23 \mathrm{sdA}$ stars observed, we clearly detect stellar pulsations in 7. Dominant pulsation periods range from 4.6 min to $12.3 \mathrm{~h}$, with most on timescales of approximately one hour. We argue specific classifications for some of the new variables, identifying both compact and likely main sequence dwarf pulsators, along with a candidate low-mass RR Lyrae star.

Conclusions. With dominant pulsation periods spanning orders of magnitude, the pulsational evidence supports the emerging narrative that the sdA class consists of multiple stellar populations. Since multiple types of sdA exhibit stellar pulsations, follow-up asteroseismic analysis can be used to probe the precise evolutionary natures and stellar structures of these individual subpopulations.
\end{abstract}

Key words. stars: oscillations - stars: variables: general - subdwarfs - white dwarfs - Galaxy: stellar content

\section{Introduction}

Model atmosphere fits to spectra from Sloan Digital Sky Survey (SDSS) data releases (DR) 10 and 12 have revealed a population of thousands of stars with H-dominated spectra, effective temperatures $T_{\text {eff }} \leq 20000 \mathrm{~K}$ (the majority with $9000 \mathrm{~K}>T_{\text {eff }}>6500 \mathrm{~K}$ ), and surface gravities $6.5>\log g>5.5$ (Kepler et al. 2015, 2016). These gravities are greater than for main sequence stars, but less than for white dwarfs (WDs) that formed through single-star evolution given the age of the Galaxy (Kilic et al. 2007). Hot sdB and sdO subdwarf stars are often observed with these gravities, but they must have $T_{\text {eff }} \gtrsim 20000$ to fuse $\mathrm{He}$ in their cores above the zero-age horizontal branch (for a recent review of subdwarfs, see Heber 2016). Extending

\footnotetext{
* Light curves of seven new pulsating variable stars are only available at the CDS via anonymous ftp to cdsarc.u-strasbg.fr (130.79.128.5) or via http://cdsarc.u-strasbg.fr/viz-bin/ qcat? J/A+A/617/A6

$\star \star$ Hubble Fellow.
}

the classification scheme for subdwarf stars, Kepler et al. (2016) applied the label "sdA" to this newly uncovered stellar population that shares an effective temperature range with main sequence A stars. This classification is based solely on spectroscopically determined parameters and does not require an evolutionary origin similar to the hot, He-burning subdwarfs. Another class of star is known to populate this $\log g-T_{\text {eff }}$ regime: extremely low-mass (ELM) WDs. The exact definition of an ELM WD varies in the literature, but they are generally understood to have degenerate $\mathrm{He}$ cores that formed as the result of mass loss in tight binaries. Interest in these objects has been spurred by recent observational discoveries and characterizations, especially by the dedicated ELM Survey (Brown et al. 2016a, and references therein). Of the 78 objects cataloged by the ELM Survey to have spectroscopic H-line profiles that agree with ELM WD atmosphere models, radial velocity variations confirm that 67 are in close binary systems (Brown et al. 2016a), supporting the binary mass-transfer formation theory. 
Despite the overlap in spectroscopic properties of the sdA and ELM WDs, the population size and other observational properties of sdA stars are not consistent with the hypothesis that they are simply older, cooler ELM WDs. Hermes et al. (2017a) appealed primarily to radial velocity variations of SDSS subspectra to argue that the vast majority of sdA stars are not in short-period binary systems, and therefore are not created through the same evolutionary pathways as the ELM Survey objects. Brown et al. (2017) consider model ELM WD evolutionary sequences (Althaus et al. 2013; Istrate et al. 2016a) to support this argument, showing that the concentration of sdA stars below $9000 \mathrm{~K}$ is too large to be consistent with further evolved ELM WDs. Comparing survey colors and reduced proper motions of the two samples, Hermes et al. (2017a) and Brown et al. (2017) both argue that most sdAs are main sequence A and F stars. Specifically, Brown et al. (2017) demonstrate that fitting main sequence model spectra with pure$\mathrm{H}$ atmosphere models can cause $\log g$ values to be systematically overestimated by up to 1 dex. By constraining the radii of six sdAs in eclipsing binaries with time series spectroscopy and Catalina Sky Survey (Drake et al. 2009) photometry, Brown et al. (2017) definitively show that at least a few specific sdA stars are too large to be cooling-track ELM WDs.

Pelisoli et al. (2018a) recently provided a thorough treatment of the problem of placing the sdA stars into a specific evolutionary context. They present the arguments for and against each of four physical interpretations:

- ELM WDs or their precursors (pre-ELM WDs): after binary interactions strip their atmospheres, He-core star models contract and then cool through the spectroscopic parameter space of sdA stars, with CNO-burning flashes possibly causing some to loop repeatedly through this space before settling onto final cooling tracks (e.g., Althaus et al. 2013; Istrate et al. 2016a). We noted above how the properties and large number of sdAs do not agree with the hotter sample of ELM Survey objects (Brown et al. 2016a).

- Blue stragglers in the halo: Brown et al. (2017) proposed that sdAs are mostly main sequence A stars with systematically overestimated $\log g$ values. Assuming main sequence radii, the spatial velocities of sdAs would place most of them in the Galactic halo, although the velocities of $35 \%$ would be too high even for this hypothesis (Pelisoli et al. 2018a). Since the main sequence lifetime of an A star is an order of magnitude shorter than the age of the halo population, these would have to be rejuvenated blue straggler stars.

- Late-type main sequence stars: dwarf stars of type F and later with $T_{\text {eff }} \lesssim 7500 \mathrm{~K}$ have main sequence lifetimes that are long enough to be consistent with the halo age. This could explain the cooler concentration of sdA stars that is revealed through density estimation of $(g-z)$ colors (Pelisoli et al. 2018a), but this supposes that spectroscopic $\log g$ values of dwarf stars are being systematically overestimated.

- Main sequence stars with hot subdwarf companions: source blending in main sequence + subdwarf binaries could yield spectroscopic $\log g$ measurements in the observed range. The main sequence star would dominate the optical flux, but the subdwarf would produce a UV excess, which Pelisoli et al. (2018a) find for only $0.5 \%$ of sdAs with GALEX photometry.

While a consensus on the exact nature of sdA stars remains out of reach, the full range of possibilities is gradually coming into focus. The main conclusion of Pelisoli et al. (2018a) is that the sdAs must consist of multiple populations in order to explain all of the observations. Pelisoli et al. (2018c) used parallaxes from Gaia DR2 to argue that hundreds of sdAs are too small to be main sequence dwarfs, but must instead be ELM WDs or their precursors. Whatever the formation channels, sdA stars represent interesting stellar populations, and better constraints on their properties will guide our theories of stellar evolution.

The potential for ELM WDs to improve our understanding of binary, mass-transfer evolution was heightened by the discovery that many exhibit photometric variations as they pulsate in a low-log $g$ extension of the ZZ Ceti (H-atmosphere WD) instability strip. Analysis of the pulsational eigenfrequencies can help to constrain the details of their interior stellar structures. We discovered the first five pulsating ELM WDs with the McDonald Observatory 2.1-m Otto Struve Telescope (Hermes et al. 2012, 2013a,b) from candidates identified in the ELM Survey. The discovery of the first pulsating ELM WD to be identified from SDSS spectra was presented in conference proceedings by Bell et al. (2015), and we include this object in the present paper. Kilic et al. (2015) published a seventh pulsating ELM WD, which is the binary companion of a millisecond pulsar.

Bell et al. (2017a) discovered three additional pulsating stars from the two most recent ELM Survey publications (Gianninas et al. 2015; Brown et al. 2016a). All three are in the region of overlap between the ELM Survey catalog and the sdAs found in SDSS spectra to have $T_{\text {eff }} \lesssim 9000 \mathrm{~K}$, and none show short-period radial velocity variations as required by some more conservative definitions of an ELM WD. Bell et al. (2017a) argue that these pulsating stars are consistent with belonging to the same population as the sdAs. One of the targets exhibits a dominant pulsation period of $4.3 \mathrm{~h}$, which exceeds the upper limit for surface reflection in a cooling-track WD (Hansen et al. 1985).

Maxted et al. (2013, 2014), Corti et al. (2016), Zhang et al. (2016), and Gianninas et al. (2016) have also reported pulsations in pre-ELM stars that evolve through the region of sdAs as they contract toward their final cooling tracks. Some of these objects pulsate at higher temperatures $\left(12000 \mathrm{~K} \gtrsim T_{\text {eff }} \gtrsim 11000 \mathrm{~K}\right)$ than a simple extrapolation of the ZZ Ceti instability strip to low $\log g$, which may be due to the contribution of atmospheric helium to the driving (Jeffery \& Saio 2013; Córsico et al. 2016; Gianninas et al. 2016; Fontaine et al. 2017). However, the claimed pulsating pre-ELMs from Corti et al. (2016) are sdA stars from SDSS spectra, and the authors suggest that the stars may be high-amplitude $\delta$ Scuti pulsators instead. While Zhang et al. (2016) claim to have observed gravity modes ( $g$-modes) in the Kepler data of a pre-ELM WD in an eclipsing binary, the relationship they find between the rotational splitting of pulsation modes and the stellar rotation rate of the star is characteristic of pressure modes ( $p$-modes). We suspect that this system is more similar to the pre-ELM WD plus pulsating $\delta$ Scuti binaries described in Zhang et al. (2017).

The many observational discoveries and measurements of pulsating stars in the parameter space of ELM and preELM WDs has prompted extensive theoretical investigations into their driving mechanisms and asteroseismic interpretations (Córsico et al. 2012, 2016; Córsico \& Althaus 2014a,b, 2016; Istrate et al. 2016b, 2017; Fontaine et al. 2017; Calcaferro et al. 2017a,b; Sánchez Arias et al. 2018).

Given the overlap in parameter space of the sdAs with the ELM WD instability strip, plus the evidence from Bell et al. (2017a) that even non-cooling-track WDs pulsate in this domain, we extended our observational search for pulsating stars at McDonald Observatory to include sdA targets. The overall timescales of sdA pulsations would give a first-order indication of the types of stars that exist in this population. Detailed followup asteroseismic interpretation of specific pulsational eigenfrequencies will go further to constrain the interior structures of these enigmatic stars. 
Table 1. Best-fit spectroscopic parameters of observed sdA stars.

\begin{tabular}{|c|c|c|c|c|c|c|}
\hline SDSS object name & Plate-MJD-Fiber & $\begin{array}{l}\text { SDSS } g \\
\text { (mag) }\end{array}$ & $\begin{array}{l}T_{\text {eff }} \\
(\mathrm{K} ; \text { solar } \mathrm{z})^{a}\end{array}$ & $\begin{array}{l}\log g \\
(\operatorname{cgs} ; \text { solar z })^{a}\end{array}$ & $\begin{array}{l}T_{\text {eff }} \\
(\mathrm{K} ; \text { pure } \mathrm{H})^{a}\end{array}$ & $\begin{array}{l}\log g \\
(\operatorname{cgs} ; \text { pure } H)^{a}\end{array}$ \\
\hline J123831.40-014654.3 & 0335-52000-0497 & 16.97 & $7932(17)$ & $4.836(0.081)$ & 7864 & 5.03 \\
\hline J131011.61-014233.0 & 0340-51990-0426 & 16.53 & $8237(10)$ & $4.721(0.060)$ & 8224 & 5.33 \\
\hline $\mathrm{J} 221524.54-005018.2$ & $0374-51791-0131$ & 17.97 & $7763(90)^{b}$ & $4.529(0.038)^{b}$ & 7838 & 5.72 \\
\hline J090804.54-000208. $8^{c}$ & $0470-51929-0076$ & 15.13 & $8168(10)$ & $5.039(0.036)$ & 8130 & 5.33 \\
\hline J091416.42+004146.8 & 0472-51955-0499 & 17.39 & $8417(20)$ & $5.883(0.081)$ & 8440 & 5.71 \\
\hline J093003.42+054815.5 & 0992-52644-0435 & 18.13 & $8760(37)$ & $6.240(0.105)$ & 8656 & 5.52 \\
\hline J074939.74+194203.5 & $1582-52939-0406$ & 18.04 & $7846(32)$ & $5.958(0.094)$ & 8042 & 6.71 \\
\hline J162953.16+220634.5 & $1658-53240-0584$ & 18.20 & $8087(35)$ & $5.833(0.122)$ & 8057 & 5.08 \\
\hline $\mathrm{J} 160410.81+062705.5$ & $1729-53858-0213$ & 15.10 & $7969(9)$ & $5.473(0.039)$ & 8097 & 5.71 \\
\hline J082900.96+084645.4 & $1758-53084-0449$ & 17.81 & $8394(25)$ & $5.995(0.100)$ & 8195 & 5.16 \\
\hline $\mathrm{J} 140119.77+351323.2$ & $1838-53467-0506$ & 17.64 & $8054(19)$ & $4.996(0.094)$ & 7908 & 5.21 \\
\hline J192253.84+783959.1 & $1857-53182-0494$ & 14.32 & $7980(10)$ & $5.328(0.032)$ & 7904 & 5.24 \\
\hline $\mathrm{J} 233258.96+490400.3$ & $1888-53239-0477$ & 16.26 & $8323(12)$ & $6.210(0.036)$ & 8283 & 5.77 \\
\hline $\mathrm{J} 223831.92+125318.3$ & $1892-53238-0249$ & 15.54 & $7980(11)$ & $4.816(0.056)$ & 7999 & 5.03 \\
\hline $\mathrm{J} 114224.61+374703.7$ & $1997-53442-0225$ & 15.09 & $8075(9)$ & $5.211(0.037)$ & 8082 & 5.16 \\
\hline J093356.45+191601.5 & $2361-53762-0308$ & 18.54 & 8333(39) & $6.088(0.127)$ & 8126 & 3.75 \\
\hline J083054.47-035118.9 & $2807-54433-0499$ & 15.15 & $8473(8)$ & $4.444(0.042)$ & 8460 & 5.03 \\
\hline J113143.46-074220.5 & $2861-54583-0078$ & 15.93 & $7853(12)$ & $4.635(0.064)$ & 7637 & 4.61 \\
\hline J045309.80-041800.7 & $3123-54741-0483$ & 16.99 & $7897(7)$ & $4.693(0.042)$ & 7665 & 4.59 \\
\hline $\mathrm{J} 074013.22+481036.7$ & $3668-55478-0370$ & 16.68 & $7961(5)$ & $3.932(0.021)$ & 7748 & 5.34 \\
\hline $\mathrm{J} 075644.33+502741.2$ & $3679-55209-0229$ & 16.56 & $7108(7)$ & $4.045(0.027)$ & 6875 & 5.34 \\
\hline $\mathrm{J} 223716.61+052228.3$ & $4291-55525-0872$ & 19.46 & $8902(40)$ & $6.695(0.017)$ & 8856 & 6.66 \\
\hline J161831.69+385415.2 & 5189-56074-0177 & 19.67 & $9307(70)$ & $7.153(0.096)$ & 9149 & 6.70 \\
\hline $\mathrm{J} 161831.69+385415.2^{d}$ & 5199-56067-0744 & 19.67 & $9354(54)$ & $6.257(0.075)$ & 9058 & 6.04 \\
\hline
\end{tabular}

Notes. ${ }^{(a)}$ We quote internal statistical uncertainties. The $T_{\text {eff }}$ and $\log g$ uncertainties for the pure-H models are similar to the solar $\mathrm{z}$ uncertainties. An analysis by Kepler et al. (2016) of the systematic uncertainties from multiple spectra indicated $5 \%$ in $T_{\text {eff }}$ and 0.05 dex in $\log g .{ }^{(b)}$ The fitting pipeline of Kepler et al. (2016) does not provide uncertainties for this fit because it does not agree with the cool $T_{\text {eff }}$ implied by the SDSS photometry for this star. We estimate the uncertainties by taking the difference between the parameters of the best fits to the tabulated Plate-MJDFiber and a second SDSS spectrum of this object, 3146-54773-0003. ${ }^{(c)}$ Eclipsing binary with 7.68-h orbit (see text). ${ }^{(d)}$ This is a second SDSS spectrum of the same object as above.

We describe the photometric observations of 23 sdA stars from McDonald Observatory, as well as the time series spectroscopic follow-up on 4 of them in Sect. 2. In Sect. 3, we present the frequency analysis of seven sdA stars that clearly exhibit pulsations in the light curves, as well as the orbital constraints from spectroscopy, studying the natures of individual objects. To conclude, we place these stars in the context of other pulsating variables in Sect. 4. We argue that the large range of dominant pulsation periods observed, from $4.6 \mathrm{~min}$. to $12.3 \mathrm{~h}$, supports that the sdAs are made up of multiple stellar populations that pulsate in this region of $\log g-T_{\text {eff }}$ space.

\section{Observations}

\subsection{Time series photometry}

We obtained $196 \mathrm{~h}$ of time series photometry on 23 stars near the low-log $g$ extension of the ZZ Ceti instability strip. Unlike the systematic search for photometric variability of ELM Survey objects described in Bell et al. (2017a), it was not practical to carry out such an exhaustive follow-up study of the thousands of sdA stars classified from SDSS spectroscopy. Our target selection was notably nonuniform, and it was often informed by parameters from preliminary fits to the SDSS spectra as we were beginning to make sense of the data. Table 1 provides the best-fit spectroscopic parameters from the two sets of models described in Pelisoli et al. (2018a): pure hydrogen atmospheres (pure H), and models matching the composition of the solar atmosphere (solar z).
The details of our observing runs are logged in Table 2. Our observational setup and data reduction pipeline was as described in Bell et al. (2017a). We observed our targets with the frametransfer ProEM CCD camera on the McDonald 2.1-m Otto Struve Telescope through a BG40 filter to reduce sky noise. All frames were dark-subtracted and flatfielded with nightly calibration frames using standard IRAF tasks. We measured circularaperture photometry to obtain light curves for the target and bright field stars with the IRAF script CCD_HSP (Kanaan et al. 2002). Then we divided each target light curve by the weighted mean comparison star light curve, clipped extreme outliers, divided low-order polynomials to remove long-timescale airmass effects, and applied a barycentric correction to the timestamps with the WQED software (Thompson \& Mullally 2013).

\subsection{Time domain spectroscopy}

The discovery of photometric variability prompted us to obtain follow-up spectroscopy of four objects, using the same instrumentation, reduction, and analysis tools as used in the ELM Survey (Brown et al. 2016a): wavelength calibration against a comparison lamp, flux calibration against blue spectrophotometric standards (Massey et al. 1988), and measurement of radial velocities (RVs) via cross-correlation with spectral templates (Kurtz \& Mink 1998).

We obtained spectra of SDSS J1618+3854 covering $3650-4500 \AA$ at $1 \AA$ resolution with the Blue Channel spectrograph (Schmidt et al. 1989) on the 6.5-m MMT. After two indi- 
Table 2. Journal of photometric observations.

\begin{tabular}{|c|c|c|c|}
\hline SDSS & $\begin{array}{c}\text { Date } \\
\text { (UTC) }\end{array}$ & $\begin{array}{l}\text { Exposure } \\
\text { Time (s) }\end{array}$ & $\begin{array}{l}\text { Run Duration } \\
\text { (h) }\end{array}$ \\
\hline \multirow[t]{11}{*}{$\mathrm{J} 1618+3854$} & 23 Apr 2014 & 20 & 6.5 \\
\hline & 24 Apr 2014 & 25 & 6.4 \\
\hline & 22 May 2014 & 30 & 1.0 \\
\hline & 22 May 2014 & 20 & 3.9 \\
\hline & 24 May 2014 & 30 & 2.6 \\
\hline & 25 May 2014 & 30 & 5.9 \\
\hline & 27 May 2014 & 30 & 7.8 \\
\hline & 21 Apr 2015 & 20 & 4.6 \\
\hline & 26 Apr 2015 & 30 & 5.7 \\
\hline & 20 May 2015 & 20 & 3.2 \\
\hline & 26 May 2015 & 30 & 7.7 \\
\hline \multirow[t]{2}{*}{ J1131-0742 } & 19 Jan 2015 & 10 & 3.7 \\
\hline & 20 Jan 2015 & 5 & 5.0 \\
\hline J0756+5027 & 11 Feb 2015 & 1 & 7.1 \\
\hline \multirow[t]{5}{*}{$\mathrm{J} 1310-0142$} & $14 \operatorname{Mar} 2015$ & 10 & 3.4 \\
\hline & 21 Jun 2017 & 15 & 1.5 \\
\hline & 21 Jun 2017 & 30 & 0.8 \\
\hline & 22 Jun 2017 & 15 & 2.6 \\
\hline & 23 Jun 2017 & 30 & 1.3 \\
\hline \multirow[t]{3}{*}{$\mathrm{J} 1142+3747$} & 25 Apr 2015 & 3 & 2.0 \\
\hline & 22 May 2017 & 15 & 3.2 \\
\hline & 24 May 2017 & 30 & 3.2 \\
\hline \multirow[t]{5}{*}{$\mathrm{J} 2238+1253$} & 11 Aug 2015 & 5 & 4.5 \\
\hline & 12 Aug 2015 & 5 & 1.6 \\
\hline & 13 Aug 2015 & 5 & 0.8 \\
\hline & 14 Aug 2015 & 3 & 5.1 \\
\hline & 16 Aug 2015 & 3 & 1.0 \\
\hline \multirow[t]{3}{*}{$\mathrm{J} 1604+0627$} & 14 Sep 2015 & 3 & 1.7 \\
\hline & 16 Sep 2015 & 5 & 2.3 \\
\hline & 06 Jul 2016 & 1 & 3.1 \\
\hline \multirow[t]{4}{*}{$\mathrm{J} 2237+0522$} & 25 Jun 2014 & 30 & 3.2 \\
\hline & 26 Jun 2014 & 30 & 3.0 \\
\hline & 12 Sep 2015 & 60 & 5.0 \\
\hline & 13 Sep 2015 & 20 & 6.1 \\
\hline J0453-0418 & 24 Jan 2015 & 10 & 2.7 \\
\hline J0830-0351 & $24 \operatorname{Jan} 2015$ & 10 & 2.5 \\
\hline $\mathrm{J} 0740+4810$ & 26 Jan 2015 & 15 & 3.6 \\
\hline $\mathrm{J} 1238-0146$ & 15 Mar 2015 & 30 & 3.3 \\
\hline J0908-0002 & 26 Apr 2015 & 3 & 2.8 \\
\hline \multirow[t]{2}{*}{$\mathrm{J} 2215-0050$} & 14 Sep 2015 & 20 & 5.2 \\
\hline & 15 Sep 2015 & 5 & 6.3 \\
\hline $\mathrm{J} 2332+4904$ & 16 Sep 2015 & 5 & 5.6 \\
\hline $\mathrm{J} 1922+7839$ & 17 Sep 2015 & 3 & 3.9 \\
\hline $\mathrm{J} 1401+3513$ & 07 Jan 2016 & 5 & 2.5 \\
\hline \multirow[t]{2}{*}{$\mathrm{J} 1629+2206$} & 06 Aug 2016 & 5 & 4.0 \\
\hline & 07 Aug 2016 & 5 & 4.7 \\
\hline \multirow[t]{3}{*}{ J0749+1942 } & 28 Nov 2016 & 10 & 1.5 \\
\hline & 28 Nov 2016 & 30 & 2.5 \\
\hline & 29 Nov 2016 & 20 & 3.1 \\
\hline \multirow[t]{2}{*}{ J0829+0846 } & 01 Jan 2017 & 10 & 2.0 \\
\hline & 02 Jan 2017 & 10 & 4.8 \\
\hline \multirow[t]{2}{*}{ J0914+0041 } & 20 Jan 2017 & 5 & 1.3 \\
\hline & 20 Jan 2017 & 5 & 1.1 \\
\hline J0933+1916 & 26 Jan 2017 & 20 & 3.7 \\
\hline $\mathrm{J} 0930+0548$ & 03 Mar 2017 & 14 & 3.0 \\
\hline
\end{tabular}

vidual observations in July 2016 and March 2017, we obtained ten additional spectra over 25-27 June 2017, and seven more on 13 May 2018.
We obtained 25 spectra of SDSS J1131-0742 during four nights between 18 February and 18 April 2015 with the FAST instrument (Fabricant et al. 1998) on the Fred L. Whipple Observatory (FLWO) 1.5-m telescope. We also obtained ten back-to-back FAST spectra of SDSS J2238+1253 over $2.1 \mathrm{~h}$ on 03 September 2016, and the same for SDSS J1142+3747 on 18 June 2017. These observations cover the wavelength range 3600-5400 A with 1.7 Åresolution.

We supplemented the observations of SDSS J1131-0742 with $2 \AA$ resolution spectra covering $3600-4950 \AA$ from the Goodman Spectrograph (Clemens et al. 2004) on the 4.1-m SOAR telescope. We obtained twelve SOAR spectra on 26 April 2016, and nine more on 31 May 2017. We followed the same basic steps to reduce these data, with a detailed description given in Pelisoli et al. (2018b).

\section{Seven new pulsating sdA stars}

The first seven sdA targets listed in Table 2 all exhibit clear pulsation signatures in our photometry. We characterize these stars individually in this section, measuring their pulsation periods and searching for RV variations from the stars that we observed spectroscopically.

All Fourier transforms (FTs) of our light curves were computed with the PERIOD04 software (Lenz \& Breger 2004) and oversampled by a factor of 20 . We followed the same process of signal detection and prewhitening described in Bell et al. (2017a), fitting and subtracting a sum of sinusoids to the most significant signals iteratively until the residuals contain no compelling peaks above a rule-of-thumb $4\langle A\rangle$ significance criterion (where $\langle A\rangle$ is the locally evaluated mean amplitude of the FT; Breger et al. 1993).

Gaps in light curves collected on multiple nights introduce aliases to our FTs that compete to describe the observed variations. Very approximately, discrete aliases for observations taken on consecutive nights have a characteristic spacing of $\approx 11.6 \mu \mathrm{Hz}$ and they span a frequency range roughly equal to the inverse of the typical run length, though actual spectral windows can be far more complicated. While the iterative prewhitening procedure can produce a frequency solution that closely matches the data, there are often many viable solutions that employ different aliases. The highest peaks in the FT do not necessarily coincide with the intrinsic signal frequencies, as Bell et al. (2017b) recently demonstrated in a comparison of ground- and space-based photometry of two pulsating WDs. We emphasize this point by exploring two candidate solutions for our first sdA pulsator, SDSS J1618+3854, below. For the other stars, we only record the frequencies of the highest amplitude aliases. The uncertainties that we quote are intrinsic to the least-squares fitting (given by analytical expressions in Montgomery \& O'Donoghue 1999); they do not account for the additional uncertainty caused by aliasing.

In our aim to assess the typical pulsation timescales of sdA targets, the tabulated frequencies are perfectly representative. Ideally, however, the full set of possible frequency solutions should be accounted for when comparing against asteroseismic models to constrain the stellar structure. This can be achieved by computing many frequency solutions through a Monte Carlo prewhitening procedure or by exploring the solution space with Markov chain Monte Carlo fitting of the light curves directly. To help with these analyses, we have published our reduced light curves for all new pulsators as supplemental material at the CDS.

For the targets that do not exhibit significant variability in our photometry, we only make limited claims about a lack of pulsations. Most were observed on a single night, and destructive 
Table 3. Limits on pulsations in sdA stars.

\begin{tabular}{|c|c|c|c|}
\hline SDSS & $\begin{array}{l}\text { Period } \\
\text { (h) }\end{array}$ & or & $\begin{array}{l}\text { Amplitude } \\
(\%)\end{array}$ \\
\hline $\mathrm{J} 2237+0522$ & $>5.0$ & & $<2.5$ \\
\hline $\mathrm{J} 2215-0050$ & $>5.2$ & & $<0.6$ \\
\hline $\mathrm{J} 1629+2206$ & $>4.0$ & & $<1.1$ \\
\hline J0749+1942 & $>3.1$ & & $<1.1$ \\
\hline J0829+0846 & $>2.0$ & & $<1.3$ \\
\hline
\end{tabular}

interference from beating of closely spaced frequencies could cause a null detection. Variations on a similar timescale to changing airmass or transparency could also mask pulsations. In Table 3 we indicate approximate limits on the periods and amplitudes of pulsations that may be present for targets that we observed for at least two hours on each of multiple nights. Following Bell et al. (2017a), we assert that each of these stars does not pulsate with periods shorter than the second-shortest run length and with amplitudes greater than the largest $4\langle A\rangle$ measured in any $200 \mu \mathrm{Hz}$ window in the FT of any nightly light curve. We note that we observed evidence of an eclipse in the light curve of SDSS J0908-0002. Photometry from the Asteroid Terrestrial-impact Last Alert System Survey (ATLAS; Heinze et al. 2018) and the Catalina Sky Survey (CSS; Drake et al. 2009) reveals eclipses and ellipsoidal variations corresponding to a 7.68-h orbit, lending support to a masstransfer evolutionary history for this object.

\subsection{SDSS J1618+3854}

The first pulsating sdA star identified from SDSS DR10 spectroscopy, SDSS J1618+3854, was presented as the sixth pulsating ELM WD in conference proceedings by Bell et al. (2015). The realization from SDSS DR12 that this may belong to the sdA population caused us to rethink this classification.

The combined light curve of the two long discovery runs on 23 and 24 April 2014 remains the easiest to interpret due to its relatively simple spectral window. Still, diurnal aliasing confuses our frequency measurements. We demonstrate the severity of this effect by presenting two of many viable frequency solutions here. A portion of the April 2014 light curve and its FT are displayed in the first row of Fig. 1.

We obtained our first frequency solution by adopting the highest peak in the FT near $203 \mu \mathrm{Hz}$ as the dominant pulsation signature. We computed a least-squares fit of a sinusoid at this frequency to the light curve and search for additional signals in the residuals. The next highest peak is near $163 \mu \mathrm{Hz}$, and we refined our fit with this sinusoid included. Continuing in this fashion, we recognized that the next three signals correspond to harmonics and combination frequencies (Brickhill 1992) of the first two signals: $2 f_{1}, f_{1}+f_{2}$, and $2 f_{1}+f_{2}$. These strict arithmetic relationships guide the choices between competing aliases, explaining our re-interpretation of the signal that the preliminary analysis of Bell et al. (2015) listed as an independent $f_{3}$. We find no additional significant signals in the residuals. We tabulate the frequencies and amplitudes of the best-fit sinusoids in Table 4 and overplot the solution in red in Fig. 1.

The dominant peak in the combined light curve from May 2014 is near $191 \mu \mathrm{Hz}$, leading us to consider a second solution to the April 2014 data that selects the second-highest alias as $f_{1}$. We prewhitened this peak and its first harmonic, which also corresponds to a peak in the FT. The highest peak in the residuals is near $227 \mu \mathrm{Hz}$. Had we not prewhitened the harmonic of $f_{1}$ first, we might have selected a different peak near $161 \mu \mathrm{Hz}$ as $f_{2}$, yielding a completely different frequency solution. After prewhitening the refined model, we adopted a third frequency near $149 \mu \mathrm{Hz}$. Finally, we included peaks corresponding to combination frequencies and harmonics, the precise locations of which justify the slight relaxation of our significance criterion. This solution is given in the bottom of Table 4 and we plot it in blue in Fig. 1.

The moral of this exercise is that one should consider exact frequency solutions from time series with gaps with skepticism. Selecting an incorrect alias can derail the prewhitening process, and tabulated solutions in the literature are usually subjective. Comparisons with asteroseismic models should try to account for this uncertainty. Alternatively, gaps can sometimes be avoided by observing from space (e.g., Hermes et al. 2017b) or from multiple sites distributed in longitude (a la the Whole Earth Telescope; Nather et al. 1990). Still, we can conclude from the present data that SDSS J1618+3854 exhibits multiple pulsation modes with periods in the $1-2 \mathrm{~h}$ range.

Encouraged by the similarity between these periods and the variability timescales of known pulsating ELM WDs (Hermes et al. 2013b), we targeted SDSS J1618+3854 for follow-up spectroscopy from the MMT. The RVs measured from two spectra obtained in July 2016 and March 2017 differed by $200 \mathrm{~km} \mathrm{~s}^{-2}$. We obtained ten additional spectra in June 2017, confirming that this is a single-lined spectroscopic binary. Seven more spectra from May 2018 improve our coverage of orbital phase, eliminating period aliases. A bootstrap analysis constrains the orbital period to $0.2664 \pm 0.0015$ days $(6.39 \pm 0.04 \mathrm{~h})$, with a semi-amplitude of $249.2 \pm 10.4 \mathrm{~km} \mathrm{~s}^{-1}$. The RV measurements folded on this period are displayed in Fig. 2. The observed photometric signals are not harmonics of the candidate RV periods, so they really are pulsations rather than signatures of a photometric binary. The orbital period is too short to permit a main sequence primary star, so we conclude that SDSS J1618+3854 is a bona fide pulsating ELM WD. Assuming an edge-on orientation, the minimum mass of the unseen companion is $0.67 M_{\odot}$.

\subsection{SDSS J1131-0742}

We selected SDSS J1131-0742 as an observing target based on excess scatter in its Catalina Sky Survey (CSS; Drake et al. 2009) photometry. Observations over two nights in January 2015 revealed dramatic peak-to-peak variations as large as $60 \%$, confirming it as a pulsating star. A segment of the light curve and the FT are displayed in the second row of Fig. 1. We list the frequencies of the highest significant peaks, revealed through successive prewhitening, in Table 5.

SDSS J1131-0742 was classified as a $\delta$ Scuti by Palaversa et al. (2013) based on data from the LINEAR survey. The two dominant modes of high-amplitude $\delta$ Scuti variables (radial fundamental and first overtone) exhibit period ratios in the range $0.76-0.80$ (e.g., Poretti et al. 2005; Pigulski et al. 2006). The period ratio of the two independent modes listed in Table 5 fall just above this range; however, there is good agreement if the intrinsic frequency for $f_{2}$ is lower by the typical daily aliasing spacing of $\sim 11.6 \mu \mathrm{Hz}$.

We obtained 25 FAST spectra and 21 SOAR spectra of SDSS J1131-0742 to search for any RV signatures of binarity. A Shapiro-Wilk test rejects the null hypothesis that the RV measurements were drawn from a Gaussian distribution with a $95 \%$ confidence level. The orbital period that minimizes $\chi^{2}$ is $4.28 \pm 0.04 \mathrm{~h}$ (with uncertainties from Monte Carlo simulations; top panel of Fig. 3). The RV semi-amplitude of $23.4 \pm 5.4 \mathrm{~km} \mathrm{~s}^{-1}$ 

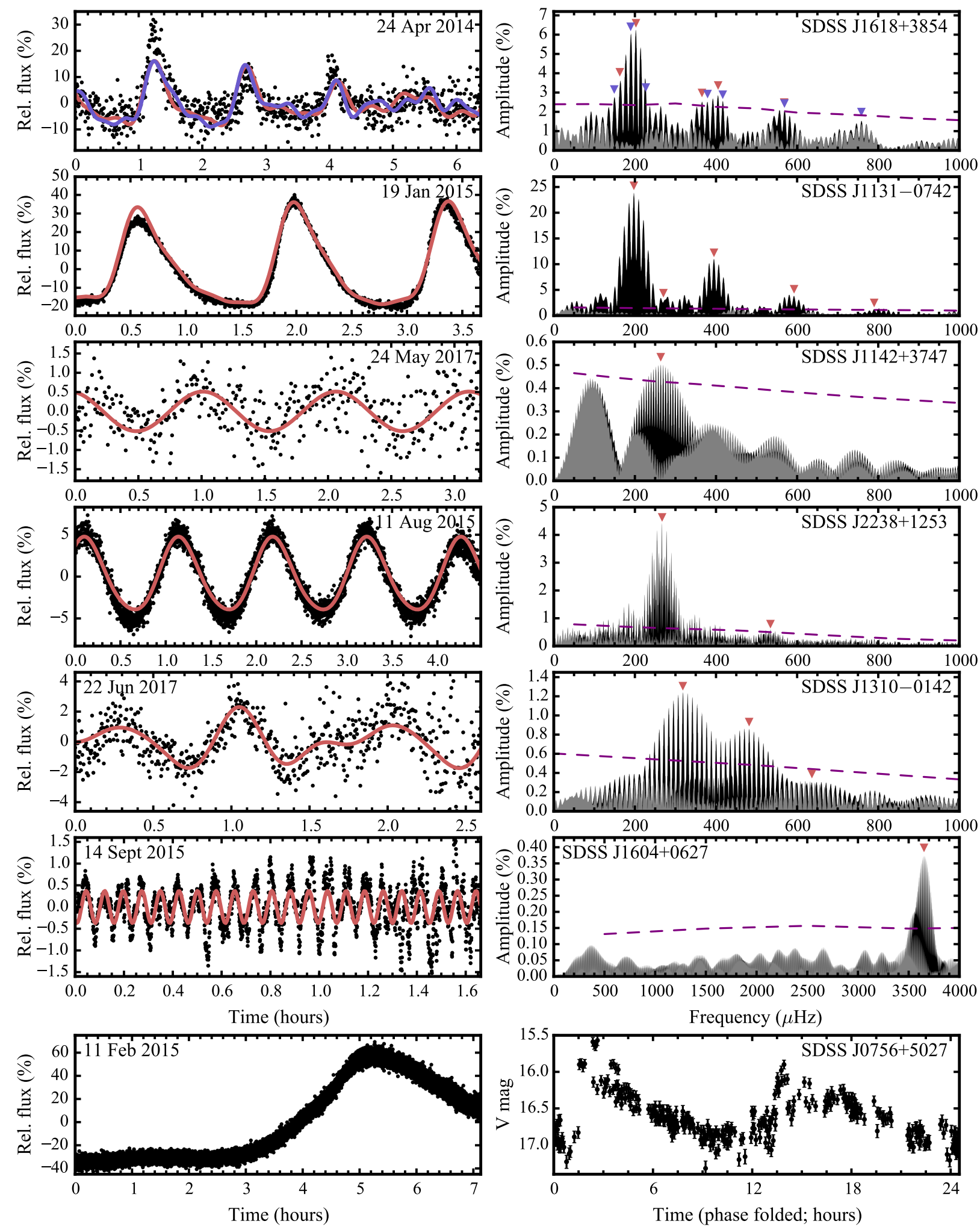

Fig. 1. Photometric signatures of pulsations in seven new pulsating sdA stars. Left panels: portions of the McDonald light curves. Top six righthand panels: FTs of data subsets (see text). Frequencies in our adopted solutions are indicated with triangles in the FTs, and the corresponding best fits are plotted over the light curves. The two frequency solutions for SDSS J1618+3854 (see text) are color-coded. Final significance thresholds are marked with dashed lines, and the FTs of the final residuals are displayed in gray. The light curve of SDSS J1604+0627 is smoothed over ten points to guide the eye. Our McDonald photometry of SDSS J0756+5027 does not cover a full pulsation period, so we plot $V$-band data from the Catalina Sky Survey (see text), folded on twice the dominant period of $12.263 \mathrm{~h}$, in the bottom right panel.

is comparable to the uncertainty on the individual measurements, and while the detection is statistically significant and phases across data from both instruments, it would be worth confirming with more precise follow-up. We plot the $\chi^{2}$ statistic against orbital period and the phase-folded RV data over this model in the bottom panel of Fig. 3. Only compact objects can fit in such 


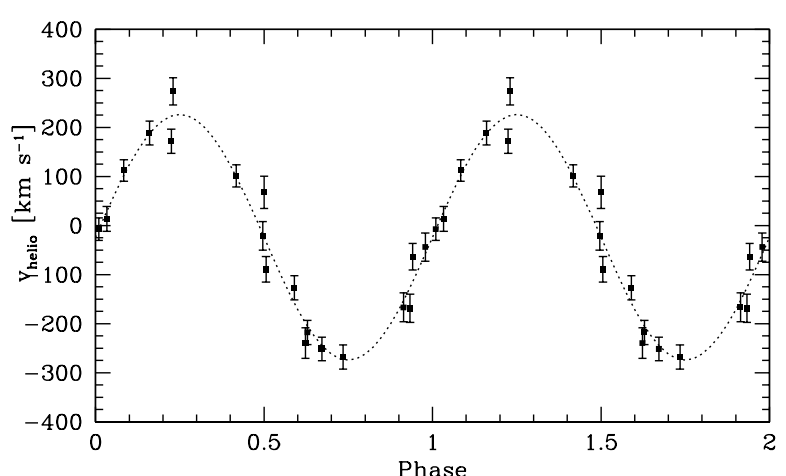

Fig. 2. Radial velocity variations of SDSS J1618 +3854 , folded on the orbital period of $6.39 \mathrm{~h}$.

Table 4. Two possible frequency solutions for the April 2014 observations of SDSS J1618+3854.

\begin{tabular}{lccr}
\hline \hline Mode ID & $\begin{array}{c}\text { Frequency } \\
(\mu \mathrm{Hz})\end{array}$ & $\begin{array}{c}\text { Period } \\
(\mathrm{s})\end{array}$ & $\begin{array}{r}\text { Amplitude } \\
(\%)\end{array}$ \\
\hline$f_{1}$ & $202.61(15)$ & $4936(4)$ & $6.19(19)$ \\
$f_{2}$ & $162.8(3)$ & $6143(11)$ & $3.35(19)$ \\
$2 f_{1}$ & $405.2(3)$ & $2468(18)$ & $2.66(19)$ \\
$f_{1}+f_{2}$ & $365.4(3)$ & $2740(20)$ & $2.68(19)$ \\
$2 f_{1}+f_{2}$ & $568.0(4)$ & $1761(12)$ & $1.88(19)$ \\
$f_{1}$ & $189.56(16)$ & $5275(5)$ & $5.63(19)$ \\
$f_{2}$ & $226.7(3)$ & $4410(7)$ & $2.76(19)$ \\
$f_{3}$ & $149.1(4)$ & $6706(16)$ & $2.59(19)$ \\
$2 f_{1}$ & $379.1(4)$ & $2638(3)$ & $2.38(19)$ \\
$f_{1}+f_{2}$ & $417.2(4)$ & $2397(2)$ & $2.21(19)$ \\
$3 f_{1}$ & $568.7(6)$ & $1758.4(17)$ & $1.69(19)$ \\
$4 f_{1}$ & $748.3(7)$ & $1318.8(12)$ & $1.37(19)$ \\
\hline
\end{tabular}

Table 5. Frequency solutions for the January 2015 observations of SDSS J1131-0742.

\begin{tabular}{lccr}
\hline \hline Mode ID & $\begin{array}{c}\text { Frequency } \\
(\mu \mathrm{Hz})\end{array}$ & $\begin{array}{c}\text { Period } \\
(\mathrm{s})\end{array}$ & $\begin{array}{r}\text { Amplitude } \\
(\%)\end{array}$ \\
\hline$f_{1}$ & $197.481(11)$ & $5063.8(3)$ & $23.43(5)$ \\
$f_{2}$ & $270.33(14)$ & $3699(2)$ & $1.86(5)$ \\
$2 f_{1}$ & $394.96(3)$ & $2531.9(2)$ & $8.71(5)$ \\
$3 f_{1}$ & $592.44(7)$ & $1687.9(2)$ & $3.62(5)$ \\
$4 f_{1}$ & $789.92(17)$ & $1265.9(3)$ & $1.56(5)$ \\
\hline
\end{tabular}

a short-period binary configuration. The minimum mass of the unseen companion is $0.021 M_{\odot}$, and it would be $0.13 M_{\odot}$ at an orbital inclination of $15^{\circ}$. With $\log g \approx 4.6$ from fits of both sets of models to the SDSS spectra, we consider this most likely to be a pulsating pre-ELM WD. We note, however, that fits of the pure-H models of Gianninas et al. (2011, 2014, and references therein) to the summed FAST spectrum yields a higher value of $\log g=5.35 \pm 0.13$.

\subsection{SDSS J1142+3747}

Our two-hour run in April 2015 revealed marginal evidence of photometric variability in SDSS J1142+3747. We revisited this object over two nights in May 2017. These data are displayed in the third row of Fig. 1.

We detect a single significant periodicity in these data, the strongest alias of which is at $263.8 \pm 0.2 \mu \mathrm{Hz}(3791 \pm 3 \mathrm{~s}$
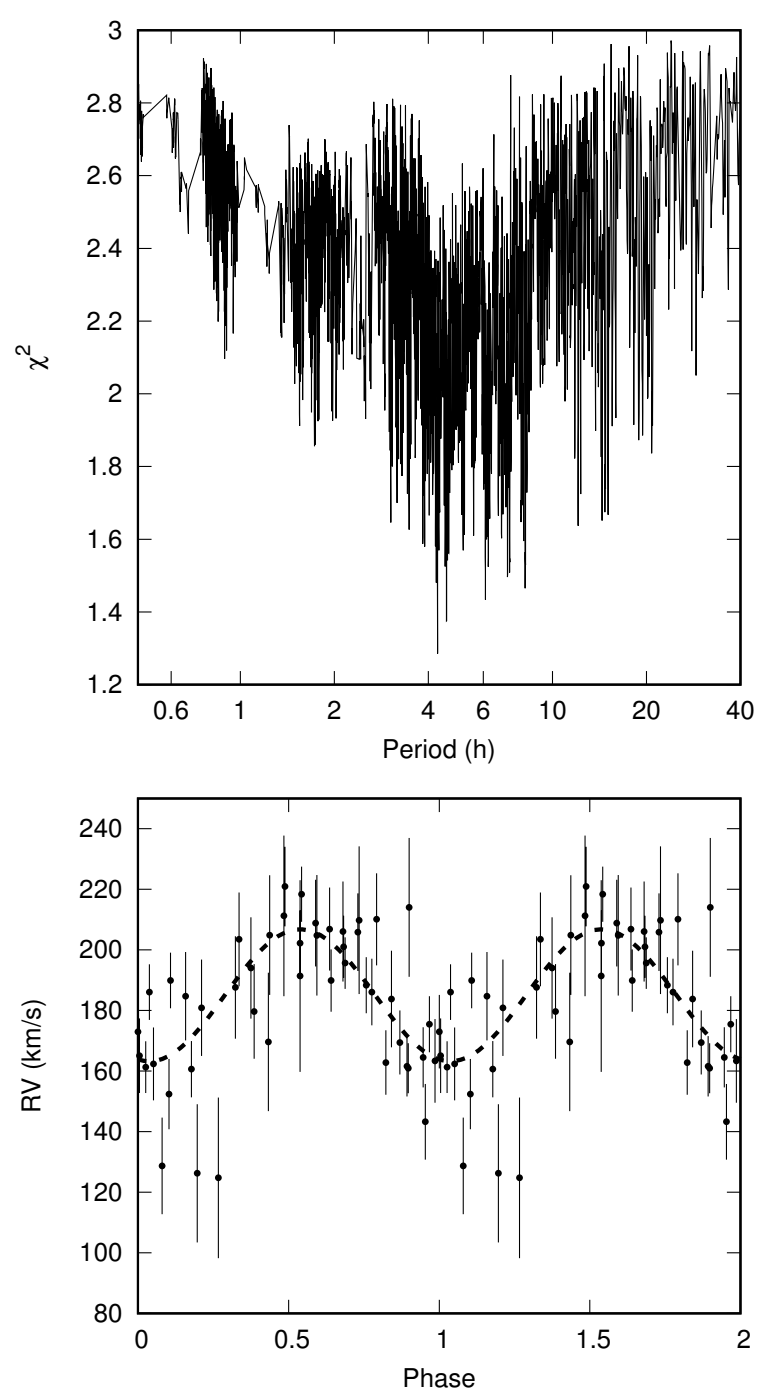

Fig. 3. Radial velocity variations of SDSS J1131-0742. Top panel: $\chi^{2}$ of fits of different periods to the RV data. Bottom panel: RV measurements folded on the best-fit period of $4.58 \mathrm{~h}$.

period) with an amplitude of $0.51 \pm 0.04 \%$. This signal timescale is consistent with pulsations observed in ELM WDs, but with only one period detected, it could also be the signature of ellipsoidal variations. ELM WDs in tight binaries often show a photometric signal at half the orbital period as the projected area of a tidally distorted star varies through the orbit (Hermes et al. 2014; Bell et al. 2017a). We obtained continuous FAST spectra over $2.1 \mathrm{~h}$-covering twice the photometric period-and found no significant RV variations to a limit of $\sim 10 \mathrm{~km} \mathrm{~s}^{-1}$. This rules against the ellipsoidal variation interpretation, supporting that SDSS J1142+3747 is a pulsating star.

sInterestingly, the high S/N summed FLWO spectrum of SDSS J1142+3747, displayed in the top panel of Fig. 4, reveals a bounty of metal lines in addition to the deep hydrogen signatures. We mark possible identifications for a few lines by comparing against the NIST Atomic Spectra Database (Kramida et al. 2018). By comparison with examples from the classification atlas of Gray (2009), SDSS J1142+3747 may be a chemically peculiar Am or Ap star (e.g., Preston 1974), some of which exhibit $\delta$ Scuti pulsations on a similar timescale (e.g., Balona et al. 2011a,b). We prefer this main sequence explanation for SDSS J1142+3747, as the spectroscopic models used to fit the SDSS data do not account for peculiar atmospheres and the inferred $\log g$ could be inaccurate as a result. 


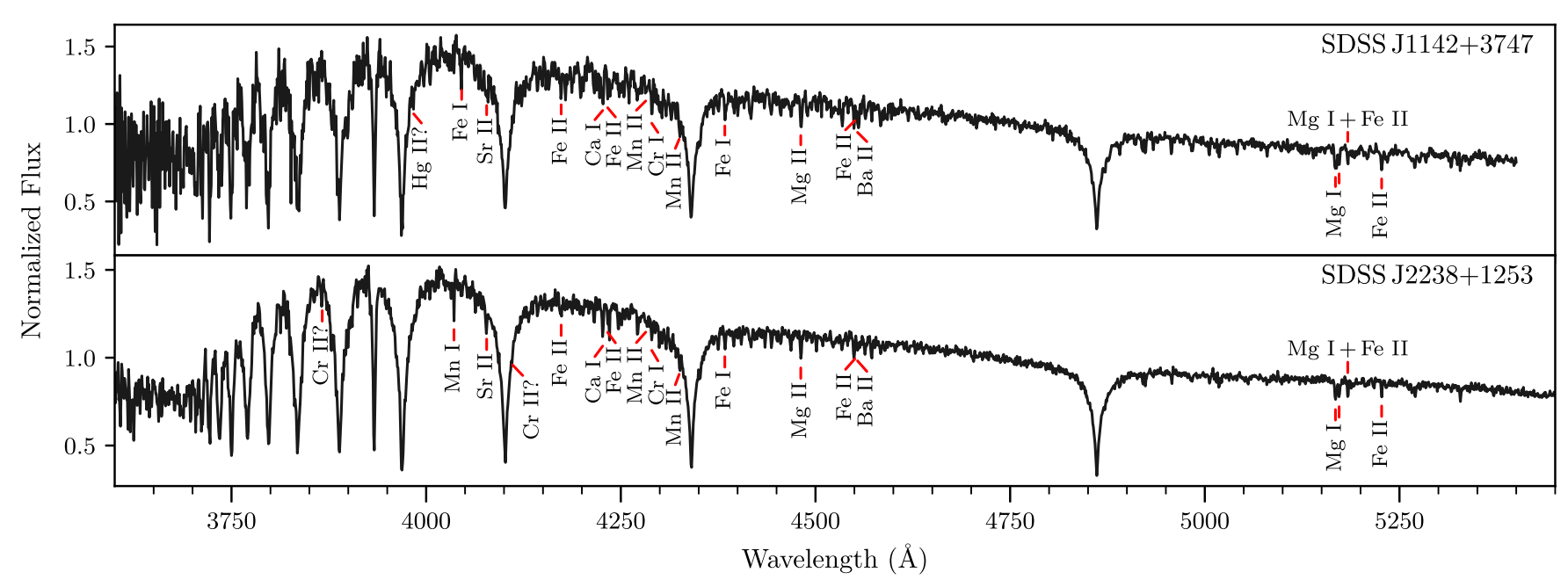

Fig. 4. Summed FAST spectra of SDSS J1142+3747 (top) and SDSS J2238+1253 (bottom) reveal many metal lines, with some candidate line identifications indicated.

\subsection{SDSS $22238+1253$}

Our analysis and interpretation of SDSS J2238+1253 follows that of the previous object, SDSS J1142+3747, very closely. This target also exhibits a single independent photometric signal with a similar period. The two peaks marked in the FT in the fourth row of Fig. 1 are the highest alias and its second harmonic, $f_{1}=267.114 \pm 0.005 \mu \mathrm{Hz}(3743.72 \pm 0.07 \mathrm{~s}$ periodicity) with an amplitude of $4.329 \pm 0.018 \%$ (harmonic amplitude at $0.488 \pm 0.018 \%$ ).

As with the previous object, we considered that a single dominant period could correspond to ellipsoidal variations, though the observed amplitude in this case is larger even than for the shortest period $(12.75 \mathrm{~min})$ detached ELM WD binary known (Brown et al. 2011). We obtained $2.1 \mathrm{~h}$ of continuous FAST spectra that do not reveal any RV variability to a limit of $\sim 10 \mathrm{~km} \mathrm{~s}^{-1}$. SDSS J2238+1253 was targeted by the parallel effort of Pelisoli et al. (2018b) to characterize sdAs with time series spectra, who also found no evidence of RV variability in the period range of $20 \mathrm{~min}$ to 180 days.

The summed FAST spectrum of SDSS J2238+1253 displayed in the bottom panel of Fig. 4 also shows many metal lines, which might be causing a blanketing effect that is unaccounted for in our atmospheric models. This may also be a chemically peculiar star (Gray 2009). We draw the same conclusion as for SDSS J1142+3747 (top panel of Fig. 4), that SDSS J2238+1253 is likely a $\delta$ Scuti pulsator on the main sequence with an overestimated $\log g$ caused by missing physics in the applied models.

\subsection{SDSS J1310-0142}

Our light curve of SDSS J1310-0142 from 14 Mar 2015 showed some evidence of photometric variability. We confirmed over three consecutive nights in June 2017 that this target is a multiperiodic pulsator. The FT and a portion of the light curve are displayed in the fifth row of Fig. 1, and the two independent signals plus a harmonic of the dominant mode are detailed in Table 6. The FT shows a broad aliasing structure, but the most likely signals do not yield a period ratio in the typical range for $\delta$ Scuti variables (0.76-0.80; e.g., Poretti et al. 2005; Pigulski et al. 2006). Sánchez Arias et al. (2018) compare pulsation periods calculated for theoretical $\delta$ Scuti and pre-ELM models to develop asteroseismic tools to distinguish these classes, but they are difficult to apply to the current observations of
Table 6. Frequency solutions for the June 2017 observations of SDSS J1310-0142.

\begin{tabular}{lccr}
\hline \hline Mode ID & $\begin{array}{c}\text { Frequency } \\
(\mu \mathrm{Hz})\end{array}$ & $\begin{array}{c}\text { Period } \\
(\mathrm{s})\end{array}$ & $\begin{array}{r}\text { Amplitude } \\
(\%)\end{array}$ \\
\hline$f_{1}$ & $318.25(15)$ & $3142.2(14)$ & $1.21(6)$ \\
$f_{2}$ & $481.7(2)$ & $2075.8(9)$ & $0.86(6)$ \\
$2 f_{1}$ & $636.5(5)$ & $1571.1(12)$ & $0.31(6)$ \\
\hline
\end{tabular}

SDSS J1310-0142 given the considerable aliasing in the spectral window. Additional future observations would be worthwhile to improve constraints on the pulsation periods and the models that can explain them.

\subsection{SDSS J1604+0627}

The photometric variability observed in SDSS J1604+0627 is quite different in character from the other new variable sdAs. The frequency of the single significant signal that we detect in this star is much higher, at $3651.35 \pm 0.19 \mu \mathrm{Hz}$ $(273.871 \pm 0.014 \mathrm{~s}$ period) with amplitude $0.37 \pm 0.02 \%$. To help make the pulsations visible to the eye, we plot a running average over ten adjacent measurements in the sixth row of Fig. 1. The displayed FT for this object runs to higher frequency in order to contain the single significant periodicity that we detect.

There are only a few known types of pulsating stars that show pulsation periods this short. The observed Balmer lines are too narrow for this to be a low-order $g$-mode in a pulsating white dwarf of typical mass. Rapidly oscillating Ap stars (roAp) exhibit short high-overtone $p$-modes, but a periodicity as short as $274 \mathrm{~s}$ has never been observed (Kurtz et al. 2006). $P$-modes in hot subdwarfs could have such short periodicities. While Pelisoli et al. (2018a) suggested that some sdAs may be binaries that contain a hot sdB star, these systems must have a UV flux excess, which we rule out for SDSS J1604+0627 given the low $($ nuv $-g$ ) color of $\approx 2.79$ from GALEX data.

Sánchez Arias et al. (2018) compare models of pre-ELM WDs that are evolving across the main sequence $(\log g \approx 4)$ to $\delta$ Scuti models and claim that periods below $700 \mathrm{~s}$ are exclusive to pre-ELM WDs. The pre-ELM models of Istrate et al. (2016b) also predicted $p$-mode pulsations with periods that agree with the 4.6-min. signal of SDSS J1604+0627. With spectroscopic 
$\log g \approx 5.7$ from fits of pure-H model atmospheres to the SDSS spectra, SDSS J1604+0627 is situated in the transition region between pre-ELM and ELM WDs in evolutionary models, and also where pre-ELM WDs may evolve repeatedly through during violent CNO-flash episodes (e.g., Althaus et al. 2013). While it has been suggested that observed pulsation periods as short as $108 \mathrm{~s}$ and $134 \mathrm{~s}$ are the signatures of $p$-modes in the pulsating ELM WD SDSS J1112+1117 (Hermes et al. 2013b), the single period observed in SDSS J1604+0627 is longer than the $p$-mode range from models of ELM WDs (Córsico \& Althaus 2016). This could instead correspond to a low- $k$ g-mode that is driven by the $\epsilon$-mechanism (Córsico \& Althaus 2014b).

Following Hermes et al. (2017a), we inspected the individual SDSS subspectra and find no evidence of RV variations on timescales of $30 \mathrm{~min}$. to 5 days, supporting that SDSS J1604+0627 is not part of a short-period binary system. Most ELM WDs in double-degenerate binaries are expected to merge within a Hubble time (Brown et al. 2016b), and a postmerger ELM WD was recently identified by Vos et al. (2018). We consider SDSS J1604+0627 to be a good candidate for the first pulsating post-merger (pre-)ELM WD.

We note, however, that it is difficult to confirm that a single periodicity is caused by pulsations. For example, in reconsidering the photometric variations in hot DQ (carbon atmosphere) WDs that were originally thought to be caused by stellar pulsations (beginning with SDSS J1426+5752; Montgomery et al. 2008), Lawrie et al. (2013) and Williams et al. (2016) have suggested that these signals can be explained more simply by fast rotational modulation. Detection of rapid rotation in SDSS J1604+0627 would also be extremely interesting in the context of a post-merger formation. Continued monitoring of SDSS J1604+0627 will help to reveal the nature of this signal as we may be able to detect the secular rate of period change from pulsations, as explored in (pre-)ELM WD evolutionary models by Calcaferro et al. (2017a). Multiwavelength observations can also discern between these explanations, as the characteristic colors of surface inhomogeneities from spots and stellar pulsations can be very different (discussed for WDs by, e.g., Dupuis et al. 2000; Robinson et al. 1982).

The unique periodicity of this sdA star will motivate continued theoretical efforts to understand the diversity of stellar pulsations and evolutionary pathways in the parameter space of sdAs. Such a short period pulsation may be sensitive to previously unprobed regions of the interior of a star in a potentially quite rare phase of evolution toward a final ELM WD cooling track.

\subsection{SDSS J0756+5027}

SDSS J0756+5027 was selected as an observing target based on significant excess scatter in the CSS data (Drake et al. 2009). Our single light curve from 11 Feb 2015 in the bottom left panel of Fig. 1 shows changes of more than a factor of two in brightness. Even with more than seven hours of coverage, we do not observe a complete period of the dominant pulsation mode.

Constructing a periodogram of the CSS photometry reveals a strong periodicity of roughly $12.263 \mathrm{~h}$, which we confirm with data from ATLAS (Heinze et al. 2018). The CSS data, folded on twice this period, is displayed in the bottom right panel of Fig. 1. Folded this way, the data suggest a change in amplitude between alternate pulsations, in line with the period doubling behavior observed in RR Lyrae stars and suspected of being connected to the Blazhko effect (Szabó et al. 2010). Drake et al. (2013) classified this star as RRab-type in the catalog of RR Lyrae from CSS data. Given the high $\log g$ inferred from pure-H model atmospheres, SDSS J0756+5027 could be physically similar to the $0.26 M_{\odot}$ star OGLE-BLG-RRLYR-02792 that exhibits RR Lyrae-like pulsations (Pietrzyński et al. 2012). That star is the recent product of mass transfer in a system that will eventually evolve to become a double-degenerate binary. Karczmarek et al. (2017) address the theoretical properties and context of such binary mass-transfer products. We note that the solar-z model fit yields a significantly lower $\log g \approx 4.05$ that approaches the typical range for RR Lyrae variables on the horizontal branch $(\log g \lesssim 3.7$; e.g., Nemec et al. 2013).

\section{Pulsational view of sdA stars}

Of the 23 sdAs observed, we find signatures of pulsations in 7. Their observational properties are summarized in Table 7. We plot the locations of all of the sdA stars that we observed in spectroscopic $\log g-T_{\text {eff }}$ space, as determined using both solar-z and pure-H models, in Fig. 5. The variables are outlined in red. We include a number of other stars for context: pulsating ELM WDs known to be in short-period binaries (Hermes et al. 2012, 2013a; Kilic et al. 2015); a pulsating pre-ELM WD in a shortperiod orbit (Maxted et al. 2014); pulsating sdA stars (not known to be in short period binaries; Hermes et al. 2013b; Corti et al. 2016; Bell et al. 2017a); and stars from the ELM Survey with limits on a lack of pulsations ( $<1 \%$ variability; some RV-variable and some not; Hermes et al. 2012, 2013a,b; Bell et al. 2017a). The empirical boundaries of the $\mathrm{H}$-atmosphere WD instability strip from Gianninas et al. (2015) are marked with dashed lines. Corrections have been applied to the spectroscopic parameters of all stars with $\log g \geq 5.0$ based on 3D hydrodynamic simulations of convection in WD atmospheres (Tremblay et al. 2013, 2015).

The pulsation timescales and orbital constraints from time series spectroscopy separate our seven new pulsating stars into several categories:

- SDSS J1618+3854 is a binary system with a 6.39-h orbital period that can only accommodate compact objects, and we also make a marginal detection of 4.58-h RV variations from SDSS J1131-0742. The pulsation periods for both are in the $1-2 \mathrm{~h}$ range, and the peak-to-peak variations are as high as $60 \%$ for SDSS J1131-0742. Given their locations in spectroscopic parameter space, the latter object may be a pre-ELM WD, while we suspect that SDSS J1618+3854 is on its final cooling track given its high spectroscopic $\log g$ compared to evolutionary models (e.g., Althaus et al. 2013; Istrate et al. 2016a).

- SDSS J1142+3747 and SDSS J2238+1253 each exhibit a single independent pulsation signal of $\sim 1 \mathrm{~h}$. We do not measure significant RV variations in their spectroscopic data, but we do detect many metal lines. We interpret these targets as possibly chemically peculiar main sequence stars that exhibit $\delta$ Scuti pulsations.

- The FT of SDSS J1310-0142 reveals two independent pulsation periods, which may be used to discern between $\delta$ Scuti and pre-ELM WD pulsations (Sánchez Arias et al. 2018) if additional observations can achieve less aliasing in the spectral window.

- SDSS J1604+0627 has a photometric period of $4.56 \mathrm{~min}$ that is unique in the sdA regime. With additional theoretical investigation, this signal may help to resolve the evolutionary state of SDSS J1604+0627, which lies in a $\log g-T_{\text {eff }}$ region of overlap of stellar models that are transitioning between contracting pre-ELM WDs and cooling-track ELM WDs, and pre-ELM WDs that are executing extremely rapid loops during episodic CNO flashes (e.g., Althaus et al. 2013; 
Table 7. Summary of new pulsating sdA star properties.

\begin{tabular}{llllll}
\hline \hline $\begin{array}{l}\text { Star } \\
(\mathrm{SDSS})\end{array}$ & $\begin{array}{l}T_{\text {eff }} \\
(\mathrm{K} ; \text { pure H) }\end{array}$ & $\begin{array}{l}\log g \\
(\mathrm{cgs} ; \text { pure H) }\end{array}$ & $\begin{array}{l}\text { Pulsation period } \\
\text { range (s) }\end{array}$ & RV variable? & suggested class \\
\hline $\mathrm{J} 1618+3854^{a}$ & 9149 & 6.70 & $5000-6100$ & yes & ELM WD \\
$\mathrm{J} 1131-0742$ & 7637 & 4.61 & $3700-5100$ & yes (?) & pre-ELM (?) \\
$\mathrm{J} 1142+3747$ & 8082 & 5.16 & 3800 & no & $\delta$ Scuti (?) \\
$\mathrm{J} 2238+1253$ & 7999 & 5.03 & 3700 & no & $\delta$ Scuti (?) \\
$\mathrm{J} 1310-0142$ & 8224 & 5.33 & $2100-3100$ & $?$ & pre-ELM/ $\delta$ Scuti (?) \\
$\mathrm{J} 1604+0627$ & 8097 & 5.71 & 274 & no & pre-ELM/ELM WD (?) \\
$\mathrm{J} 0756+5027$ & 6875 & 5.34 & 44100 & $?$ & ELM WD/RR Lyrae (?) \\
\hline
\end{tabular}

Notes. ${ }^{(a)}$ See Table 1 for another set of spectroscopic parameters.

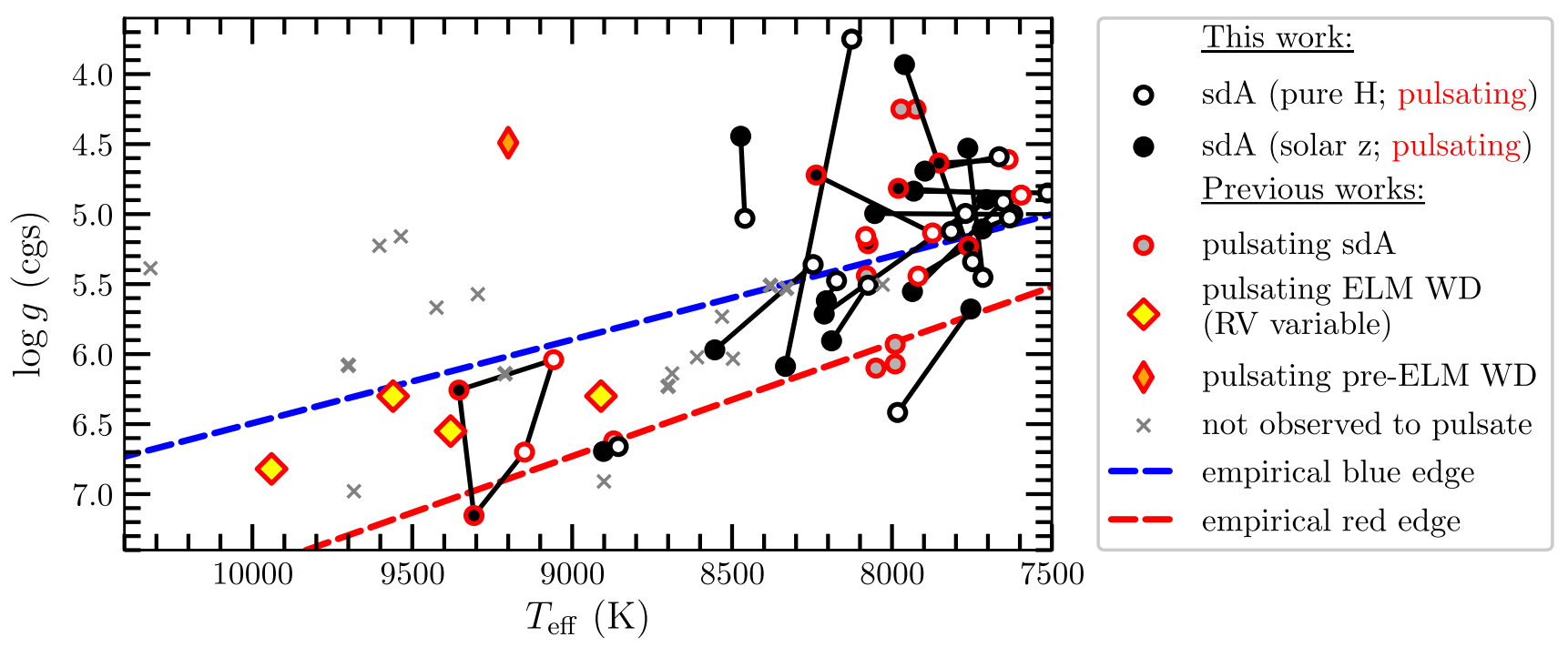

Fig. 5. Spectroscopic parameters from pure-H (white filled circles) and solar-z (black filled circles) model fits to SDSS spectra are connected by lines for 23 sdA stars that we observed photometrically. We fit two spectra of SDSS J1618+3854, represented by four connected points in this figure. New pulsating variables are outlined in red. We indicate other variables and non-variables from previous searches for pulsating (pre-)ELM WDs for context, along with the empirical low-mass extension of the ZZ Ceti instability strip boundaries (see text).

Istrate et al. 2016a). SDSS subspectra indicate that this star is not in a short-period binary, so it may be a post-merger object.

- The dominant pulsation period of SDSS J0756+5027 is $12.3 \mathrm{~h}$, and we interpret from the light curve morphology that this star is undergoing RR Lyrae-type pulsations. It may be a pre-ELM WD like the low-mass RR Lyrae-type variable OGLE-BLG-RRLYR-02792 (Pietrzyński et al. 2012).

The diversity in pulsation properties of the variable sdA stars supports the emerging narrative that this population comes from a mixture of formation and evolution scenarios. It also reveals that it is an asteroseismically rich population, with many of the subclasses exhibiting pulsations. More detailed follow-up analyses of the observed pulsational eigenfrequencies will enable us to constrain the interior structures of these stars, including the products of mass transfer in tight binaries.

SDSS J1618+3854 is an important addition to the pulsating ELM WD class that has been growing steadily since the first discovery by Hermes et al. (2012). Based on the preliminary characterization of SDSS J1618+3854 presented by Bell et al. (2015), Calcaferro et al. (2017b) found that the pulsation periods of SDSS J1618+3854 agree best with calculations for an ELM WD model with $M_{\star}=0.1706 M_{\odot}$ and $T_{\text {eff }}=9076 \mathrm{~K}$. Bell et al. (2015) suggested that the nonlinearities in the pulsations of SDSS J1618+3854 make it a good candidate to asteroseismically constrain the behavior of stellar convection in the new physical regime of ELM WDs, following the method of Montgomery
(2005). We maintain that this would be a valuable analysis, but it requires that we pin down the intrinsic pulsation frequencies from among the aliases, which will likely require an observing campaign using multiple sites.

Pre-ELMs represent an exciting phase of stellar evolution, where many stars might execute extremely rapid CNOflash loops that burn residual hydrogen (those with masses $0.18 M_{\odot} \lesssim M_{\mathrm{WD}} \lesssim 0.4 M_{\odot}$ in the models of Althaus et al. 2013). The ELM WD precursors can be quite large before they reach the cooling track; e.g., Pietrzyński et al. (2012) constrain the radius of the low-mass RR Lyrae-type variable OGLE-BLG-RRLYR02792 to be $>4 R_{\odot}$ from eclipses. Three of our new variables exhibit exotic variations that may provide particular insight into pulsational driving and interior structures in the pre-ELM WD regime. The high-amplitude pulsations of SDSS J1131-0742, the 4.56-min. period of SDSS J1604+0627, and the RR Lyraetype variability of SDSS J0756+5027 all warrant additional theoretical investigations to be sufficiently explained. Existing theoretical diagnostics to asteroseismically discern between pre-ELM WDs and $\delta$ Scuti variables from Sánchez Arias et al. (2018) insist that the short period observed in SDSS J1604+0627 cannot belong to a $\delta$ Scuti star.

Preliminary constraints on sdA radii from Gaia DR2 parallaxes indicate that hundreds are ELM WDs or their precursors (Pelisoli et al. 2018c). Additional analyses and data from future Gaia releases will further disentangle the properties of the 
multiple sdA subpopulations. Independent radius measurements for pulsating sdAs and ELM WDs will also inform our asteroseismic constraints on stellar structures, which is especially important for stars that exhibit only a few pulsation modes, such as those presented here.

In parallel and in agreement with the present work, Pelisoli et al. (2018b) detected pulsations with $\sim 1-2 \mathrm{~h}$ periods in time series photometry of 7 out of 21 additional sdA stars that they observed.

To enable more detailed asteroseismic analyses, we have published the reduced light curves of all new variable sdAs as a supplement to this manuscript.

Acknowledgements. We thank the referee for helpful comments that improved this manuscript. We thank Alex Gianninas for fitting models to the FAST spectrum of SDSS J1131-0742, Michel Breger for many helpful discussions about $\delta$ Scuti variables, and Arumalla B. S. Reddy for assistance in identifying meta lines in FAST spectra. We also thank Alejandro Córsico, Leila Calcaferro, and Leandro Althaus for inspecting the pulsation properties of their CNO-flashing evolutionary models. K.J.B., D.E.W., K.I.W., Z.V., B.G.C., and M.H.M. acknowledge support from NSF grant AST-1312983. K.J.B. was also supported by the European Research Council under the European Community's Seventh Framework Programme (FP7/2007-2013)/ERC grant agreement no. 338251 (StellarAges) during the preparation of this manuscript. I.P. acknowledges support from CNPQ-Brazil. Support for this work was provided by NASA through Hubble Fellowship grant \#HST-HF2-51357.001-A, awarded by the Space Telescope Science Institute, which is operated by the Association of Universities for Research in Astronomy, Inc., under NASA contract NAS5-26555. A preliminary version of this work was included in K.J.B.'s PhD thesis (U. Texas). This paper includes data taken at The McDonald Observatory of The University of Texas at Austin. Thanks go to the McDonald observing support, especially David Doss, Coyne Gibson, and John Kuehne. The authors acknowledge the Texas Advanced Computing Center (TACC) at The University of Texas at Austin for providing database resources that have contributed to the research results reported within this paper. The CSS survey is funded by the National Aeronautics and Space Administration under Grant No. NNG05GF22G issued through the Science Mission Directorate Near-Earth Objects Observations Program. The CRTS survey is supported by the U.S. National Science Foundation under grants AST-0909182 and AST-1313422.

\section{References}

Althaus, L. G., Miller Bertolami, M. M., \& Córsico, A. H. 2013, A\&A, 557, A19 Balona, L. A., Cunha, M. S., Kurtz, D. W., et al. 2011a, MNRAS, 410, 517 Balona, L. A., Ripepi, V., Catanzaro, G., et al. 2011b, MNRAS, 414, 792

Bell, K. J., Kepler, S. O., Montgomery, M. H., et al. 2015, in 19th European Workshop on White Dwarfs, 493, 217

Bell, K. J., Gianninas, A., Hermes, J. J., et al. 2017a, ApJ, 835, 180

Bell, K. J., Hermes, J. J., Vanderbosch, Z., et al. 2017b, ApJ, 851, 24

Breger, M., Stich, J., Garrido, R., et al. 1993, A\&A, 271, 482

Brickhill, A. J. 1992, MNRAS, 259, 519

Brown, W. R., Kilic, M., Hermes, J. J., et al. 2011, ApJ, 737, L23

Brown, W. R., Gianninas, A., Kilic, M., Kenyon, S. J., \& Allende Prieto, C. 2016a, ApJ, 818, 155

Brown, W. R., Kilic, M., Kenyon, S. J., \& Gianninas, A. 2016b, ApJ, 824, 46

Brown, W. R., Kilic, M., \& Gianninas, A. 2017, ApJ, 839, 23

Calcaferro, L. M., Córsico, A. H., \& Althaus, L. G. 2017a, A\&A, 600, A73

Calcaferro, L. M., Córsico, A. H., \& Althaus, L. G. 2017b, A\&A, 607, A33

Clemens, J. C., Crain, J. A., \& Anderson, R. 2004, Proc. SPIE, 5492, 331

Córsico, A. H., \& Althaus, L. G. 2014a, A\&A, 569, A106

Córsico, A. H., \& Althaus, L. G. 2014b, ApJ, 793, L17

Córsico, A. H., \& Althaus, L. G. 2016, A\&A, 585, A1

Córsico, A. H., Romero, A. D., Althaus, L. G., \& Hermes, J. J. 2012, A\&A, 547, A96

Córsico, A. H., Althaus, L. G., Serenelli, A. M., et al. 2016, A\&A, 588, A74

Corti, M. A., Kanaan, A., Córsico, A. H., et al. 2016, A\&A, 587, L5

Drake, A. J., Djorgovski, S. G., Mahabal, A., et al. 2009, ApJ, 696, 870

Drake, A. J., Catelan, M., Djorgovski, S. G., et al. 2013, ApJ, 763, 32

Dupuis, J., Chayer, P., Vennes, S., Christian, D. J., \& Kruk, J. W. 2000, ApJ, 537 977

Fabricant, D., Cheimets, P., Caldwell, N., \& Geary, J. 1998, PASP, 110, 79
Fontaine, G., Istrate, A., Gianninas, A., Brassard, P., \& Van Grootel, V. 2017, in 20th European White Dwarf Workshop, 509, 347

Gianninas, A., Bergeron, P., \& Ruiz, M. T. 2011, ApJ, 743, 138

Gianninas, A., Dufour, P., Kilic, M., et al. 2014, ApJ, 794, 35

Gianninas, A., Kilic, M., Brown, W. R., Canton, P., \& Kenyon, S. J. 2015, ApJ, 812,167

Gianninas, A., Curd, B., Fontaine, G., Brown, W. R., \& Kilic, M. 2016, ApJ, 822, L27

Gray, R. O. 2009, A Digital Spectral Classification Atlas (Pasadena: IPAC); ned. ipac. caltech.edu/level5/Gray/

Hansen, C. J., Winget, D. E., \& Kawaler, S. D. 1985, ApJ, 297, 544

Heber, U. 2016, PASP, 128, 082001

Heinze, A. N., Tonry, J. L., Denneau, L., et al. 2018, ApJ, submitted [arXiv:1804.02132]

Hermes, J. J., Montgomery, M. H., Winget, D. E., et al. 2012, ApJ, 750, L28

Hermes, J. J., Montgomery, M. H., Winget, D. E., et al. 2013a, ApJ, 765, 102

Hermes, J. J., Montgomery, M. H., Gianninas, A., et al. 2013b, MNRAS, 436, 3573

Hermes, J. J., Brown, W. R., Kilic, M., et al. 2014, ApJ, 792, 39

Hermes, J. J., Gänsicke, B. T., \& Breedt, E. 2017a, 20th European White Dwarf Workshop, 509, 453

Hermes, J. J., Gänsicke, B. T., Kawaler, S. D., et al. 2017b, ApJS, 232, 23

Istrate, A. G., Marchant, P., Tauris, T. M., et al. 2016a, A\&A, 595, A35

Istrate, A. G., Fontaine, G., Gianninas, A., et al. 2016b, A\&A, 595, L12

Istrate, A. G., Fontaine, G., \& Heuser, C. 2017, ApJ, 847, 130

Jeffery, C. S., \& Saio, H. 2013, MNRAS, 435, 885

Kanaan, A., Kepler, S. O., \& Winget, D. E. 2002, A\&A, 389, 896

Karczmarek, P., Wiktorowicz, G., Iłkiewicz, K., et al. 2017, MNRAS, 466, 2842

Kepler, S. O., Pelisoli, I., Koester, D., et al. 2015, MNRAS, 446, 4078

Kepler, S. O., Pelisoli, I., Koester, D., et al. 2016, MNRAS, 455, 3413

Kilic, M., Stanek, K. Z., \& Pinsonneault, M. H. 2007, ApJ, 671, 761

Kilic, M., Hermes, J. J., Gianninas, A., \& Brown, W. R. 2015, MNRAS, 446 L26

Kramida, A., Ralchenko, Yu., Reader, J., \& NIST ASD Team. 2018, NIST Atomic Spectra Database (ver. 5.5.6), https://physics.nist.gov/asd [2018, April 13]

Kurtz, M. J., \& Mink, D. J. 1998, PASP, 110, 934

Kurtz, D. W., Elkin, V. G., Cunha, M. S., et al. 2006, MNRAS, 372, 286

Lawrie, K. A., Burleigh, M. R., Dufour, P., \& Hodgkin, S. T. 2013, MNRAS, 433, 1599

Lenz, P., \& Breger, M. 2004, The A-Star Puzzle, 224, 786

Massey, P., Strobel, K., Barnes, J. V., \& Anderson, E. 1988, ApJ, 328, 315

Maxted, P. F. L., Serenelli, A. M., Miglio, A., et al. 2013, Nature, 498, 463

Maxted, P. F. L., Serenelli, A. M., Marsh, T. R., et al. 2014, MNRAS, 444, 208

Montgomery, M. H. 2005, ApJ, 633, 1142

Montgomery, M. H., \& Odonoghue, D. 1999, Delta Scuti Star Newsletter, 13, 28 Montgomery, M. H., Williams, K. A., Winget, D. E., et al. 2008, ApJ, 678, L51

Nather, R. E., Winget, D. E., Clemens, J. C., Hansen, C. J., \& Hine, B. P. 1990, ApJ, 361, 309

Nemec, J. M., Cohen, J. G., Ripepi, V., et al. 2013, ApJ, 773, 181

Palaversa, L., Ivezić, Ž., Eyer, L., et al. 2013, AJ, 146, 101

Pelisoli, I., Kepler, S. O., \& Koester, D. 2018a, MNRAS, 475, 2480

Pelisoli, I., Kepler, S. O., Koester, D., et al. 2018b, MNRAS, 478, 867

Pelisoli, I., Bell, K. J., Kepler, S. O., \& Koester, D. 2018c, MNRAS, submitted [arXiv:1805.04070]

Pietrzyński, G., Thompson, I. B., Gieren, W., et al. 2012, Nature, 484, 75

Pigulski, A., Kołaczkowski, Z., Ramza, T., \& Narwid, A. 2006, Mem. Soc. Astron. It., 77, 223

Poretti, E., Suárez, J. C., Niarchos, P. G., et al. 2005, A\&A, 440, 1097

Preston, G. W. 1974, ARA\&A, 12, 257

Robinson, E. L., Kepler, S. O., \& Nather, R. E. 1982, ApJ, 259, 219

Sánchez Arias, J. P., Romero, A. D., Córsico, A. H., et al. 2018, A\&A, 616, A80

Schmidt, G. D., Weymann, R. J., \& Foltz, C. B. 1989, PASP, 101, 713

Szabó, R., Kolláth, Z., Molnár, L., et al. 2010, MNRAS, 409, 1244

Thompson, S., \& Mullally, F. 2013, Astrophys. Source Code Lib., ascl:1304.004

Tremblay, P.-E., Ludwig, H.-G., Steffen, M., \& Freytag, B. 2013, A\&A, 559, A104

Tremblay, P.-E., Gianninas, A., Kilic, M., et al. 2015, ApJ, 809, 148

Vos, J., Zorotovic, M., Vučković, M., Schreiber, M. R., \& Østensen, R. 2018, MNRAS, 477, L40

Williams, K. A., Montgomery, M. H., Winget, D. E., Falcon, R. E., \& Bierwagen, M. 2016, ApJ, 817, 27

Zhang, X. B., Fu, J. N., Li, Y., Ren, A. B., \& Luo, C. Q. 2016, ApJ, 821, L32

Zhang, X. B., Fu, J. N., Liu, N., Luo, C. Q., \& Ren, A. B. 2017, ApJ, 850, 125 